\title{
An unusual cause of polymorphic ventricular tachycardia: Acute cervical cord injury.
}

Jogendra Singh ${ }^{1}$, Debasish das ${ }^{1}$, Debasis Acharya ${ }^{2}$, Ramachandra Barik ${ }^{2}$, Dibya Behera ${ }^{2}$, and Dibya Mahanta ${ }^{2}$

${ }^{1}$ All India Institute of Medical Sciences - Bhubaneswar

${ }^{2}$ All India Institute of Medical Sciences Bhubaneswar

January 18, 2021

\begin{abstract}
Cardiovascular disturbances are the leading causes of morbidity and mortality in patients of spinal cord particularly cervical cord injury accounting for approximately $30 \%$ of deaths. Most common cardiovascular dysfunctions are sinus bradycardia, hypotension, cardiac arrest, supraventricular tachycardia and all these occurs due to sympathetic withdrawal and unopposed vagal action. Here we are reporting a case of acute cervical cord injury with neurogenic shock in a 25 year young patient who developed polymorphic ventricular tachycardia, which degenerated to ventricular fibrillation and cardiac arrest. We described all possible mechanisms of development this arrythmia and its management.
\end{abstract}

An unusual cause of polymorphic ventricular tachycardia: Acute cervical spinal cord injury.

Jogendra Singh MBBS, MD. Debasish das MBBS, MD, PDF. Debasis acharya MBBS, MD, DM, PDF. Rama Chandra Barik MBBS, MD, DNB. Dibya Ranjan Behera MBBS, MD, DM. Dibya Sundar Mahanta MBBS, MD.

Department of cardiology, All india institute of medical sciences, Bhubaneswar, Odisha, India 751019.

Correspondence-

Dr Debasis Acharya, Department of cardiology, All India Institute of Medical Sciences. Bhubaneswar, Odisha, India 751019.

Email:debasisacharyabhu@gmail.com

Mob No- +917675992616

Disclosures: None

Funding: None

KEYWORDS:

Polymorphic ventricular tachycardia

QTc interval

Autonomic imbalance

Heart rate variability 


\begin{abstract}
Cardiovascular disturbances are the leading causes of morbidity and mortality in patients of spinal cord particularly cervical cord injury accounting for approximately $30 \%$ of deaths. Most common cardiovascular dysfunctions are sinus bradycardia, hypotension, cardiac arrest, supraventricular tachycardia and all these occurs due to sympathetic withdrawal and unopposed vagal action. Here we are reporting a case of acute cervical cord injury with neurogenic shock in a 25 year young patient who developed polymorphic ventricular tachycardia, which degenerated to ventricular fibrillation and cardiac arrest. We described all possible mechanisms of development this arrythmia and its management.
\end{abstract}

Introduction - Cardiovascular disturbances are the leading causes of morbidity and mortality in patients of spinal cord particularly cervical cord injury. Cardiovascular dysfunctions are common cause of death, accounting for approximately $30 \%$ of deaths (1). Most common cardiovascular dysfunctions are sinus bradycardia, hypotension, supraventricular tachycardia rarely cardiac arrest. All these occurs due to sympathetic withdrawal and unopposed vagal action (2). However polymorphic ventricular tachycardia has not been described in these patients. Here we are reporting a case of acute cervical spinal cord injury with neurogenic shock in a 25 year young patient who developed polymorphic ventricular tachycardia degenerating to ventricular fibrillation and cardiac arrest. We described all possible mechanisms of development this arrythmia and its management

Case- A 25 year old male was admitted to emergency room with history of road traffic accident. On arrival he was in shock with pulse rate $65 / \mathrm{min}$, systolic blood pressure of $60 \mathrm{~mm}$ of $\mathrm{Hg}$. He was in respiratory distress with type 1 respiratory failure. He was intubated, started inotropes dopamine and shifted to radiology department for imaging of spine. Imaging revealed fracture dislocation of cervical vertebra at level C5 and C6 with injury to cervical cord at that level (Fig-1). So a diagnosis of acute cervical spinal cord injury with neurogenic shock was made and planned for spinal cord decompression and fixation of fractured and dislocated cervical vertebra. Electrocardiogram (ECG) showed sinus rhythm with heart rate of around 100 per minute and QTc interval of $448 \mathrm{~m} \mathrm{sec}($ Fig-2). Screening echocardiography showed structurally normal heart. Patient developed intermittent sinus bradycardia for which atropine was given. For refractory hypotension midodrine was advised and after 2 hours of giving first dose of midodrine $10 \mathrm{mg}$ through ryles tube, he developed sudden polymorphic ventricular tachycardia (Fig-3). This VT degenerated to ventricular fibrillation and cardiac arrest. Immediately cardiopulmonary resuscitation was done and patient was revived. Then he developed accelerated idioventricular rhythm transiently (Fig-4). Injectable magnesium sulphate was given. All electrolytes like sodium, potassium magnesium and calcium were found to be normal. Intravenous lidocaine was started. ECG post event showed normal QTc interval of $445 \mathrm{~m} \mathrm{sec} \mathrm{(}$ Fig-5). After stabilisation patient was taken for cervical spinal cord decompression and fixation. Subsequent ECG had normal range of QTc interval. Patient was discharged in a stable condition after few days.

\title{
Discussion-
}

Spinal cord injury particularly above T6 vertebra level cause disruption of sympathetic system and unopposed vagal stimulation which can lead to several effects on CV system. Major effects are persistent bradycardia, neurogenic shock, rarely asystole. Some patients develop atrial fibrillation and flutter. Patients with partial spinal cord disruption may sometimes develop autonomic dysreflexia which is characterised by sudden increase in blood pressure in response to triggering events (2). Most common dysrhythmia in acute cervical cord injury is persistent sinus bradycardia which peaks at day 4 and generally resolves at 4 to 6 weeks. Rarely patients develop severe bradycardia and cardiac arrest. Ventricular tachycardia has not been described in spinal cord injury patient in literature but may develop if patient has certain risk factor like previous cardiac disease or if patient develops stress induced cardiomyopathy or electrolyte imbalance or on multiple QT prolonging drugs. Our patient had no history of any significant illness, structurally normal heart during event and on discharge, and was not on any QT prolonging drugs.

Polymorphic ventricular tachycardia is uncommon and can occur in setting of normal or increased QT interval (3). Polymorphic VT in setting of normal QT interval occurs in acute myocardial ischemia, brugada syndrome, catecholaminergic PVT and electrolyte imbalance, digitalis toxicity and hypertrophic cardiomyopathy (4). Polymorphic VT in setting of increased QT interval is a special category called Torsades de 
pointes and has acquired or congenital causes. This distinction is important because treatment options for both categories are different. Polymorphic VT with normal QT interval can be treated with lidocaine or amiodarone whereas amiodarone is contraindicated in second category. Intravenous magnesium sulfate, isoprenaline infusion and rapid pacing at rate of 100 to 120 with temporary pacemaker to decrease QT interval are treatment options in torsades.

Bunten D C et al have shown that Heart rate variability (HRV) is altered in cervical spinal cord trauma patients and may have prognostic significance (5). Further Hillebrand Stefanie et al have shown than loss of HRV is associated with increased risk of cardiovascular events in population with no cardiovascular disease (6). So autonomic imbalance along with loss of heart rate variability may be triggering factors in initiation of polymorphic VT in our patient.

\section{Conclusion-}

Acute cervical spinal cord injury is common and have important cardiovascular system consequences. Although very rare, still patients may develop polymorphic ventricular tachycardia, fibrillation and cardiac arrest. Critical care physician must be vigilant enough to recognise this form of tachycardia. Distinction between polymorphic VT with normal QT and polymorphic VT with QT prolongation is of paramount importance as treatment protocols differ. Autonomic imbalance along with loss of heart rate variability may be triggering factors in initiation of this arrythmia.

\section{Figure Legends-}

Figure 1. Imaging showing Fracture dislocation at level C5 and C6 vertebra with injury to cervical cord at that level.

Figure 2 Electrocardiogram showing sinus rhythm, heart rate of 100 per minute and QTc interval of $448 \mathrm{~m}$ sec.

Figure 3. Apparently normal QTc interval during sinus rhythm, R on T phenomenon followed by initiation of polymorphic ventricular tachycardia later degenerating to ventricular fibrillation.

Figure 4 showing sinus rhythm followed by few fusion complexes and idioventricular rhythm after resuscitation.

Figure 5 ECG just after event showing Sinus rhythm with QTc of $445 \mathrm{~m} \mathrm{sec}$.

\section{Figures-}

\section{Fig-1}




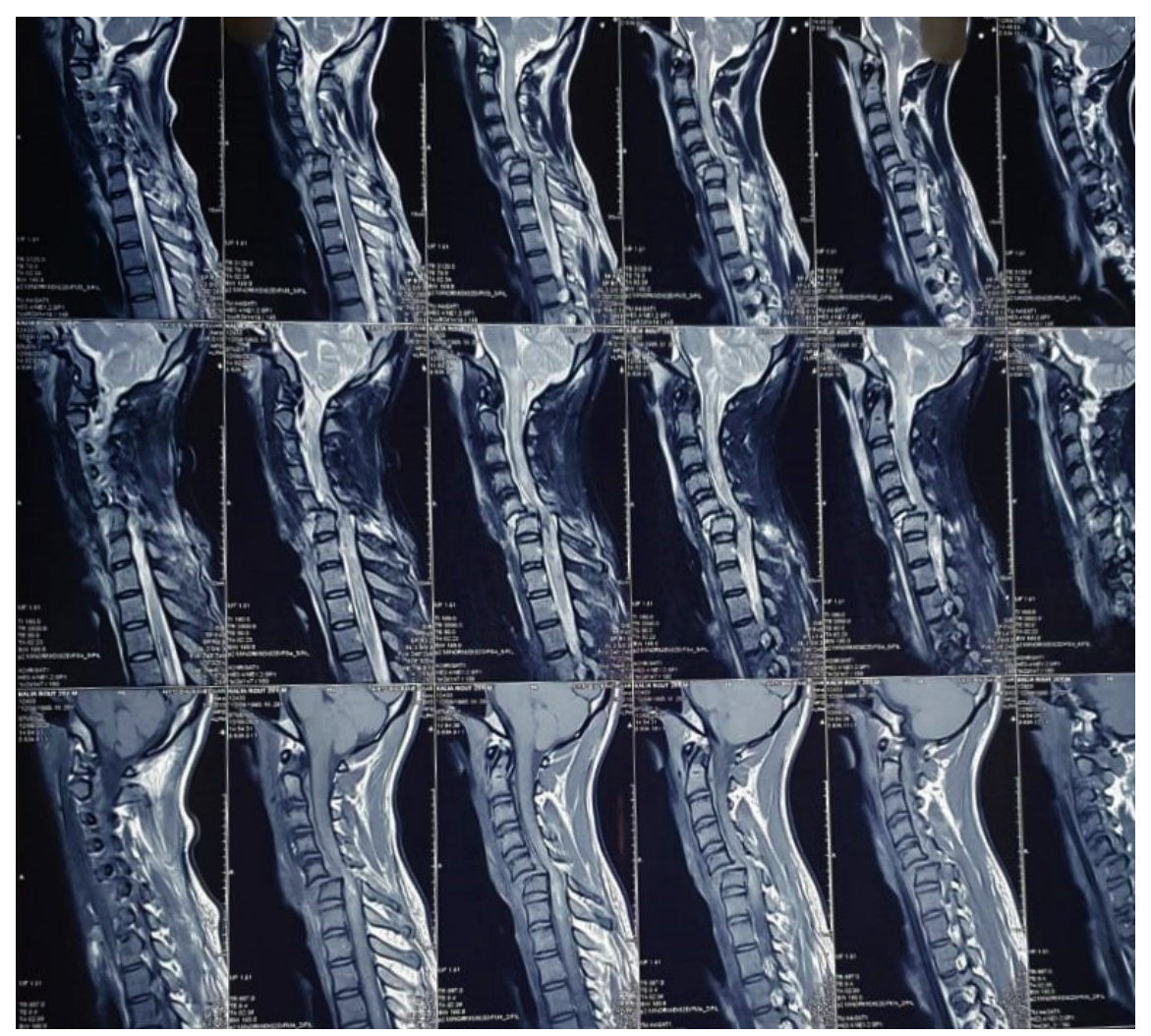

Figure 1. Imaging showing Fracture dislocation at level C5 and C6 vertebra with injury to cervical cord at that level.

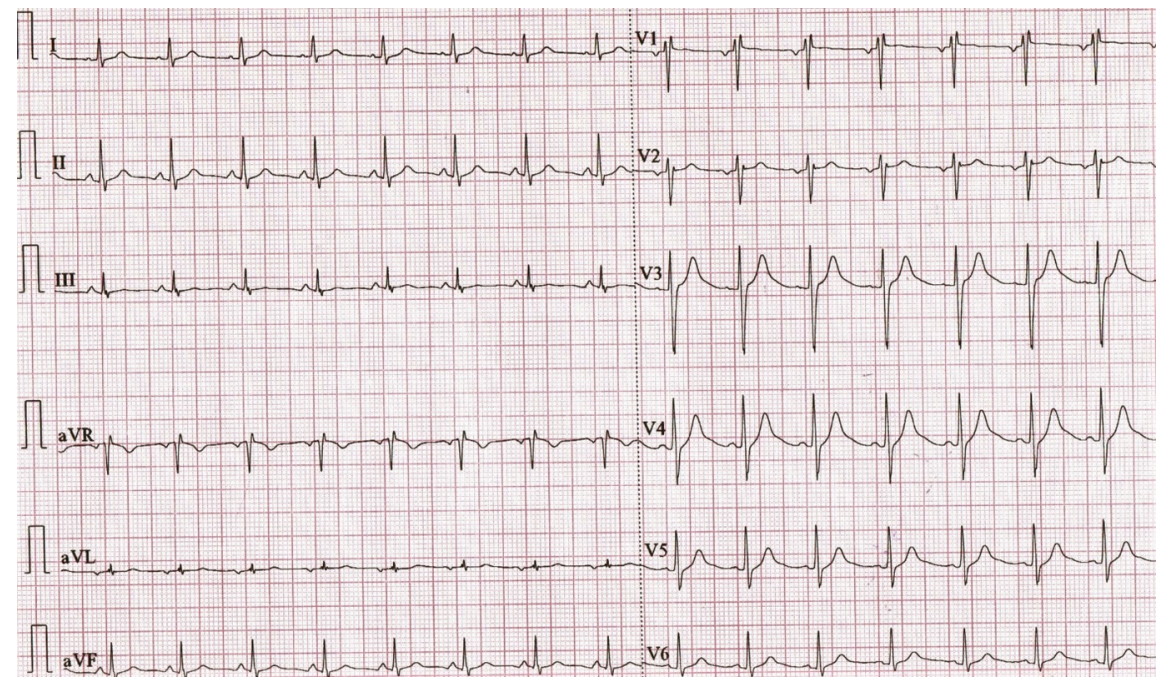

\section{Fig-2}

Figure 2 Electrocardiogram showing sinus rhythm, heart rate of 100 per minute and QTc interval of $448 \mathrm{~m}$ sec.

Fig-3 


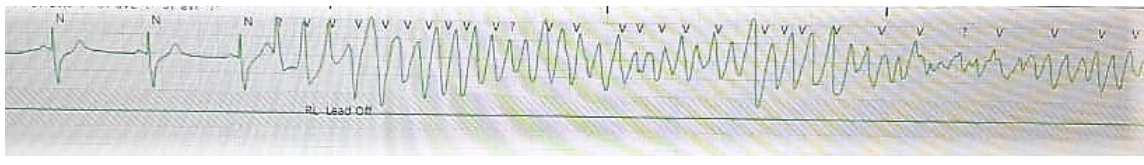

Figure 3. Apparently normal QTc interval during sinus rhythm, R on $\mathrm{T}$ phenomenon followed by initiation of polymorphic ventricular tachycardia later degenerating to ventricular fibrillation.

Fig-4

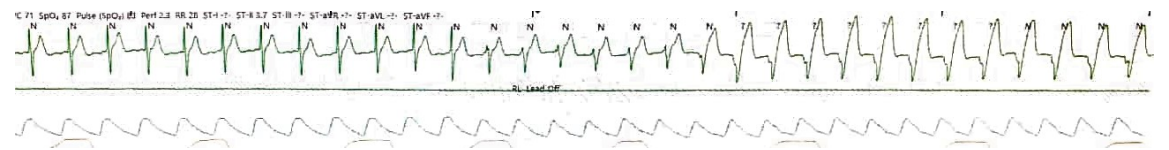

Figure 4 showing sinus rhythm followed by few fusion complexes and idioventricular rhythm after resuscitation.

Fig-5

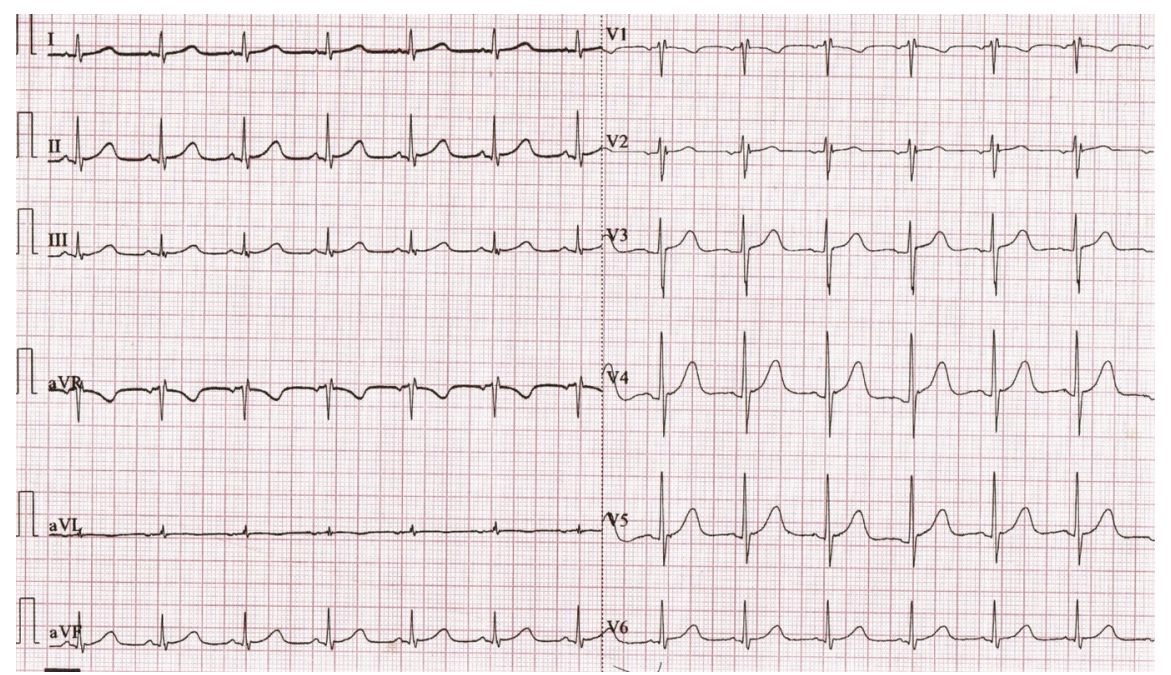

Figure 5 ECG just after event showing Sinus rhythm with QTc of $445 \mathrm{~m} \mathrm{sec}$

\section{References-}

1. Garshick E, Kelley A, Cohen SA et al, A prospective assessment of mortality in chronic spinal cord injury. Spinal Cord 43[7], 408- 416. 2005.

2. Valentine Titus Gregorean, Aurelia Mihaela Sandu, Mihai Popescu et al. Cardiac dysfunctions following spinal cord injury. Journal of Medicine and Life, Vol 2, No.2, April-june 2009, pp.133-145.

3. Passmann R, Kadish A. Polymorphic ventricular tachycardia, long QT syndrome and torsades de pointes. Med Clin North Am. 2001;85:321-341.

4. Lim W, Chiamvimonvat N, Amsterdam EA. Electrogram with a twist. Critical Pathw Cardio. 2012;11:218-219.

5. Bunten D C, Warner AL, Brunnemann S R et al. Heart rate variability is altered following spinal cord injury. Clin Auton Res. 1998 Dec;8(6)329-334.

6. Hillebrand S, Gast K,Mutsert R et al. Heart rate variability and first cardiovascular event in populations without known cardiovascular disease; A metaanalysis. EP Europace May 2013;15( 5):p742-749. 